\title{
Lattice-based Equation of State of QCD matter with a critical point
}

\author{
Claudia Ratti* \\ Department of Physics, University of Houston \\ E-mail: crattiauh.edu
}

\begin{abstract}
We build a family of Equations of State (EoS) for QCD, which match the lattice QCD results up to $\mathscr{O}\left(\mu_{B}^{4}\right)$, and contain a critical point in the 3D Ising model universality class. We place the critical point in the chemical potential range covered by the second Beam Energy Scan (BESII) at the Relativistic Heavy Ion Collider (RHIC). We show the effect of the critical point on several thermodynamic observables and discuss possible constraints on the parameters, which arise from mapping the Ising model phase diagram onto the QCD one.
\end{abstract}

Corfu Summer Institute 2018 "School and Workshops on Elementary Particle Physics and Gravity" (CORFU2018)

31 August - 28 September, 2018

Corfu, Greece

\footnotetext{
* Speaker.
} 


\section{Introduction}

The main purpose of the second Beam Energy Scan at RHIC is to discover the critical point on the QCD phase diagram, which separates the known crossover transition at low chemical potential $\mu_{B}$ [1] from a postulated first order phase transition at larger density/chemical potential. A first principle prediction of the existence and location of the critical point is still missing, due to the fermionic sign problem that hinders Monte Carlo simulations at finite chemical potentials. However, the existence of the critical point has been predicted by several QCD-like models (for a review see e.g. [2]). The location of the critical point depends on the model used to predict it, which makes its experimental search very challenging. In view of the BESII, it is therefore important to predict the behavior of experimental observables in the vicinity of the critical point.

Hydrodynamical simulations have proven to be extremely successful in describing the matter created in heavy-ion collisions. Even if hydrodynamics itself needs to be modified in the vicinity of a critical point $[3,4,5,6]$, the equation of state which serves as an input to these simulations must contain a critical point with the correct singular behavior, besides reproducing all known constraints from lattice QCD. We have recently constructed a family of equations of state, which match lattice QCD results up to $\mathscr{O}\left(\mu_{B}^{4}\right)$ and contain a critical point in the 3D Ising model universality class [7].

At $\mu_{B}=0$, the EoS of QCD is known with high precision, in the case of $2+1[8,9,10]$ and $2+1+1$ [11] quark flavors. Lattice QCD simulations allow us to reconstruct the equation of state as a Taylor series in powers of $\mu_{B} / T[12,13,14,15,16]$ or an analytic continuation from imaginary $\mu_{B}[17,18,19,20,21,22,23,24]$. The Taylor expansion of the pressure in $\mu_{B} / T$ around $\mu_{B}=0$ can be written as:

$$
P\left(T, \mu_{B}\right)=T^{4} \sum_{n} c_{2 n}(T)\left(\frac{\mu_{B}}{T}\right)^{2 n}
$$

where the coefficients of the expansion are the susceptibilities of the baryon number:

$$
c_{n}(T)=\left.\frac{1}{n !} \frac{\partial^{n} P / T^{4}}{\partial\left(\mu_{B} / T\right)^{n}}\right|_{\mu_{B}=0}=\frac{1}{n !} \chi_{n}(T) .
$$

After the early results for $c_{2}, c_{4}$ and $c_{6}$ [13], the first continuum extrapolated results for $c_{2}$ were published in Ref. [25]; in Ref. [26] $c_{4}$ was shown, but only at finite lattice spacing. The continuum limit for $c_{6}$ was published for the first time in [27], and later in [28]. In [29], a first determination of $c_{8}$, at two values of the temperature and $N_{t}=8$ was presented. More recently, an estimate of the temperature dependence of $c_{8}$ was presented at $N_{t}=12$ [30]. The advantage of the Taylor expansion method is that all the quantities are calculated at vanishing baryon chemical potential, where lattice QCD simulations do not suffer from the fermion sign problem (for a recent review, see e.g. [31]).

\section{Ising model Equation of State}

The parameterization of the scaling Equation of state for the Ising model is usually given in terms of magnetization $M$ as a function of magnetic field $h$ and reduced temperature $r=\left(T-T_{c}\right) / T_{c}$ (or auxiliary variables $R$ and $\theta$. The following form for the parametrization meets the requirements 
$[32,33,34,35]:$

$$
\begin{aligned}
M & =M_{0} R^{\beta} \theta, \\
h & =h_{0} R^{\beta \delta} \tilde{h}(\theta), \\
r & =R\left(1-\theta^{2}\right) .
\end{aligned}
$$

where $M_{0}, h_{0}$ are normalization constants, $\tilde{h}(\theta)=\theta\left(1+a \theta^{2}+b \theta^{4}\right)$ with $a=-0.76201, b=$ 0.00804. $\beta \simeq 0.326$ and $\delta \simeq 4.80$ are 3D Ising critical exponents, and the parameters take on the values $R \geq 0,|\theta| \leq \theta_{0} \simeq 1.154, \theta_{0}$ being the first non-trivial zero of $\tilde{h}(\theta)$. The values of the normalization constants are such that $M\left(r=-1, h=0^{+}\right)=1$ and $M(r=0, h) \propto \operatorname{sgn}(h)|h|^{1 / \delta}$ : this yields $M_{0} \simeq 0.605, h_{0} \simeq 0.394$.

The Gibbs free energy density then follows from this parametrization:

$$
G(h, r)=F(M, r)-M h,
$$

where $F(M, r)$ is the free energy density, defined as:

$$
F(M, h)=h_{0} M_{0} R^{2-\alpha} g(\theta),
$$

where $\alpha \simeq 0.11$ is another critical exponent of the 3D Ising model (also, the relation $2-\alpha=\beta(\delta+$ 1 ) holds). The function $g(\theta)$ is fixed by noticing that $h=(\partial F / \partial M)_{h}$ and solving the following differential equation:

$$
\tilde{h}(\theta)\left(1-\theta^{2}+2 \beta \theta^{2}\right)=2(2-\alpha) \theta g(\theta)+\left(1-\theta^{2}\right) g^{\prime}(\theta)
$$

which results in:

$$
g(\theta)=c_{0}+c_{1}\left(1-\theta^{2}\right)+c_{2}\left(1-\theta^{2}\right)^{2}+c_{3}\left(1-\theta^{2}\right)^{3}
$$

with:

$$
\begin{aligned}
& c_{0}=\frac{\beta}{2-\alpha}(1+a+b), \\
& c_{1}=-\frac{1}{2} \frac{1}{\alpha-1}\{(1-2 \beta)(1+a+b)-2 \beta(a+2 b)\}, \\
& c_{2}=-\frac{1}{2 \alpha}\{2 \beta b-(1-2 \beta)(a+2 b)\}, \\
& c_{3}=-\frac{1}{2(\alpha+1)} b(1-2 \beta) .
\end{aligned}
$$

To proceed with the mapping of the Ising model onto the QCD phase diagram we notice that the Gibbs free energy density equals the pressure up to a minus sign: $G=-P$, hence:

$$
P_{\text {Ising }}(R, \theta)=h_{0} M_{0} R^{2-\alpha}[\theta \tilde{h}(\theta)-g(\theta)] .
$$

We now map the phase diagram of the 3D Ising model onto the one of QCD, so that the critical point of the Ising model $r=h=0$ corresponds to the one of $\mathrm{QCD}$, and that the lines of first order phase transition and crossover in the Ising model are mapped onto those of QCD. 
The simplest way to do so is through a linear map as follows [36]:

$$
\begin{aligned}
\frac{T-T_{C}}{T_{C}} & =w\left(r \rho \sin \alpha_{1}+h \sin \alpha_{2}\right), \\
\frac{\mu_{B}-\mu_{B C}}{T_{C}} & =w\left(-r \rho \cos \alpha_{1}-h \cos \alpha_{2}\right),
\end{aligned}
$$

which can be visualized in Fig. 1. This map makes use of six parameters, two of which correspond to the location of the critical point on the QCD phase diagram, two are the angles that the $r$ and $h$ axes form with the $T=$ const. lines, and $(w, \rho)$ are scale factors for the variables $r$ and $h$. While $w$ represents a global scaling for the Ising variables, namely determining the size of the critical region, $\rho$ represents a relative scaling of $r$ and $h$, thus roughly determining the shape of it.
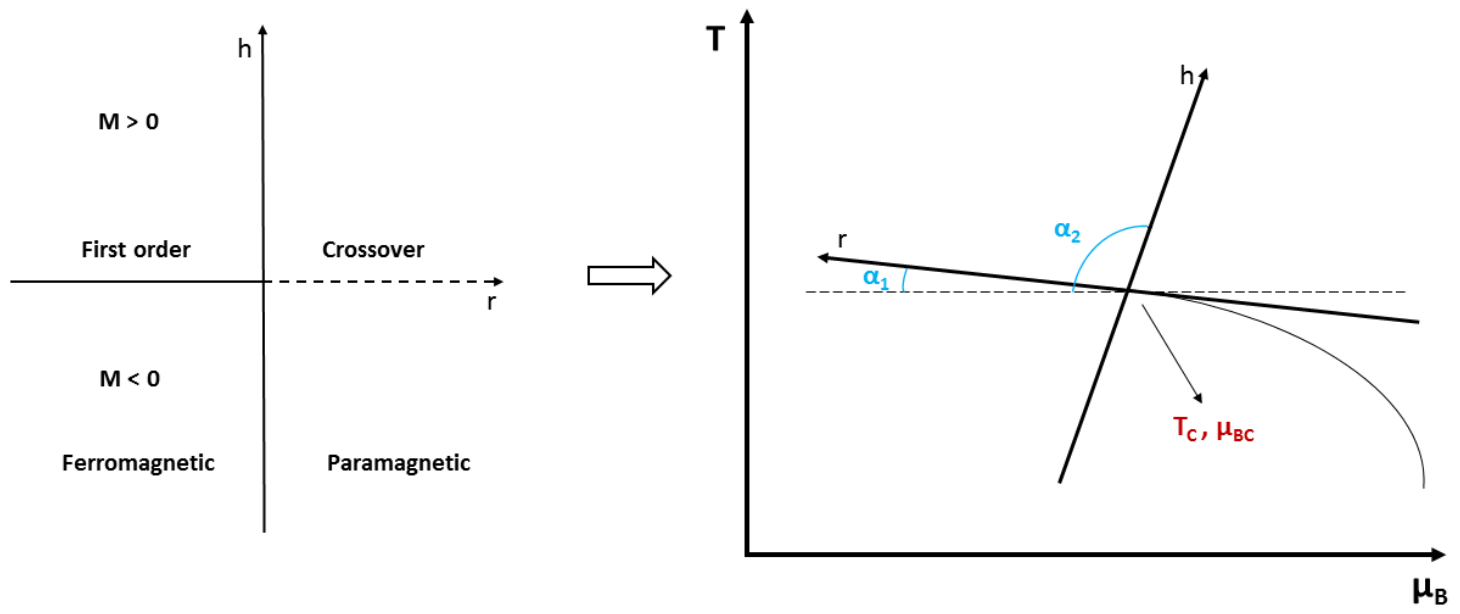

Figure 1: Non-universal map from Ising variables $(r, h)$ to $\mathrm{QCD}$ coordinates $\left(T, \mu_{B}\right)$

At this point it is possible to transport the thermodynamics of the Ising model (written in terms of $(R, \theta)$ ), into the QCD phase diagram, given a choice of parameters for the map.

It is possible to impose some constraint on the parameter choice by making use of additional arguments for the location of the critical point. For example, the curvature of the transition line at $\mu_{B}=0$ has been estimated in lattice simulations [37, 38, 39]. The shape of such transition line can be approximated with a parabola:

$$
T=T_{0}+\kappa T_{0}\left(\frac{\mu_{B}}{T_{0}}\right)^{2}+\mathscr{O}\left(\mu_{B}^{4}\right)
$$

where $T_{0}$ and $\kappa$ are the transition temperature and curvature of the transition line at $\mu_{B}=0$, respectively. The number of the parameters is thus reduced to four, the angle $\alpha_{1}$ also being fixed by:

$$
\alpha_{1}=\tan ^{-1}\left(2 \frac{\kappa}{T_{0}} \mu_{B C}\right) .
$$

In the following, remembering that the aim of the EoS is to be employed in hydrodynamic simulations for heavy-ion collisions in the BES-II program, we will consider a choice of the baryonic 
chemical potential which is $\mu_{B C}=350 \mathrm{MeV}$, resulting in:

$$
T_{C} \simeq 143.2 \mathrm{MeV}, \quad \alpha_{1} \simeq 3.85^{\circ} .
$$

In addition, the axes are chosen to be orthogonal, as we already mentioned, so that $\alpha_{2} \simeq 93.85^{\circ}$. Finally, the scaling parameters are initially chosen as:

$$
w=1, \quad \rho=2 .
$$

Later we will explore different choices for $w$ and $\rho$, trying to reduce their acceptable range on the basis of physical conditions for the thermodynamic quantities.

\section{Thermodynamics}

In the following, we assume that the lattice QCD expansion coefficients can be written as a sum of an "Ising" contribution coming from the critical point of QCD, and a "Non-Ising" contribution, which would contain the regular part as well as any other possible criticality present in the region of interest:

$$
T^{4} c_{n}^{\mathrm{LAT}}(T)=T^{4} c_{n}^{\mathrm{Non}-\mathrm{Ising}}(T)+f\left(T, \mu_{B}=0\right) c_{n}^{\mathrm{Ising}}(T) .
$$

where $f\left(T, \mu_{B}\right)$ is a regular function of the temperature and chemical potential, with dimension of energy to the fourth power. We choose $f\left(T, \mu_{B}\right)=T_{C}^{4}$. Note that $\mathrm{Eq}$ (3.1) is to be understood as a definition for the $c_{n}^{\text {Non-Ising }}$ coefficients, which we obtain as a difference between the other two contributions. The full pressure is then reconstructed simply by adding the critical contribution at any $\left(T, \mu_{B}\right)$ to the Taylor expanded "Non-Ising" one:

$$
P\left(T, \mu_{B}\right)=T^{4} \sum_{n} c_{2 n}^{\text {Non-Ising }}(T)\left(\frac{\mu_{B}}{T}\right)^{2 n}+P_{\text {crit }}^{\mathrm{QCD}}\left(T, \mu_{B}\right) .
$$

Note that in Eq. (3.2), the critical pressure is obtained from Eq. (2.12) with the multiplication by $f\left(T, \mu_{B}\right)$ in Eq. (3.1):

$$
P_{\mathrm{QCD}}^{\mathrm{crit}}\left(T, \mu_{B}\right)=f\left(T, \mu_{B}\right) P^{\mathrm{Ising}}\left(R\left(T, \mu_{B}\right), \theta\left(T, \mu_{B}\right)\right) .
$$

Because of the charge conjugation symmetry, in QCD the partition function needs to be an even function of the baryon chemical potential:

$$
\mathscr{Z}\left(T,-\mu_{B}\right)=\mathscr{Z}\left(T, \mu_{B}\right),
$$

as well as the pressure. Thus QCD must possess a critical point at both $\mu_{B C}$ and $-\mu_{B C}$. To achieve this we need to write Eq. (3.5) below. This form does not modify the singular critical behavior at the critical point(s) and automatically ensures that the odd-power coefficients in the Taylor expansion in $\mu_{B}$ vanish, as they should.

$$
\begin{aligned}
P_{\mathrm{QCD}}^{\mathrm{crit}}\left(T, \mu_{B}\right) & =\frac{1}{2} f\left(T, \mu_{B}\right) P_{\mathrm{symm}}^{\mathrm{Ising}}\left(R\left(T, \mu_{B}\right), \theta\left(T, \mu_{B}\right)\right)= \\
& =\frac{1}{2} f\left(T, \mu_{B}\right)\left\{P^{\mathrm{Ising}}\left(R\left(T, \mu_{B}\right), \theta\left(T, \mu_{B}\right)\right)+P^{\mathrm{Ising}}\left(R\left(T,-\mu_{B}\right), \theta\left(T,-\mu_{B}\right)\right)\right\}
\end{aligned}
$$


which will have the effect of slightly changing the form of the critical pressure (the main one being that now the pressure at the critical point is non-zero, whereas it would be zero in the straightforward definition) but not its singular behavior, leaving all the even order derivatives unchanged.

In Fig. 2 we can see the comparison between the lattice data (and the extension with the HRG model) and the resulting parametrization. The HRG model employed to calculate the pressure does not contain any interaction, and makes use of the most up to date particle list available from the Particle Data Group [40] (list PDG2016+ in [41]).

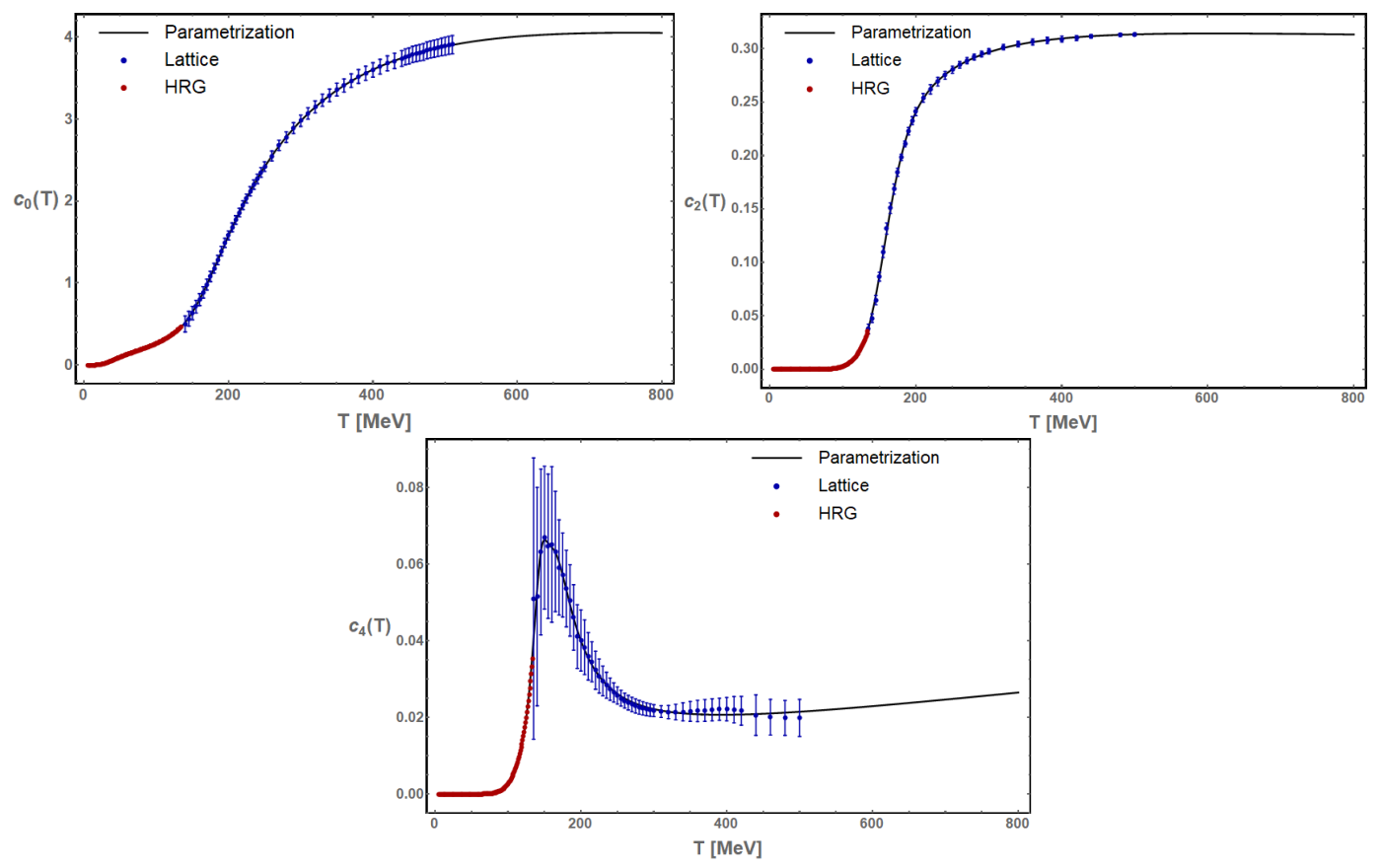

Figure 2: Parametrization of baryon susceptibilities from Lattice QCD [9, 42] and HRG model calculations.

Fig. 3 shows the comparison of the "Ising" and "Non-Ising" contributions to the parametrized lattice/HRG model results.

\section{Results}

To cure some pathological behavior of our EoS at small temperatures, we perform a smooth merging with the HRG model EoS.

The smooth merging can be obtained through a hyperbolic tangent as:

$$
\begin{aligned}
\frac{P_{\mathrm{Final}}\left(T, \mu_{B}\right)}{T^{4}}= & \frac{P\left(T, \mu_{B}\right)}{T^{4}} \frac{1}{2}\left(1+\tanh \left(\frac{T-T^{\prime}\left(\mu_{B}\right)}{\Delta T^{\prime}}\right)\right)+ \\
& +\frac{P_{\mathrm{HRG}}\left(T, \mu_{B}\right)}{T^{4}} \frac{1}{2}\left(1-\tanh \left(\frac{T-T^{\prime}\left(\mu_{B}\right)}{\Delta T^{\prime}}\right)\right),
\end{aligned}
$$

where $T^{\prime}\left(\mu_{B}\right)$ works as the "switching temperature", and $\Delta T^{\prime}$ is roughly the size of the "overlap region" where both pressures contribute to the sum. The dependence on the baryon chemical potential of the "switching temperature" is chosen to be parabolic: this way, the "switching line" 

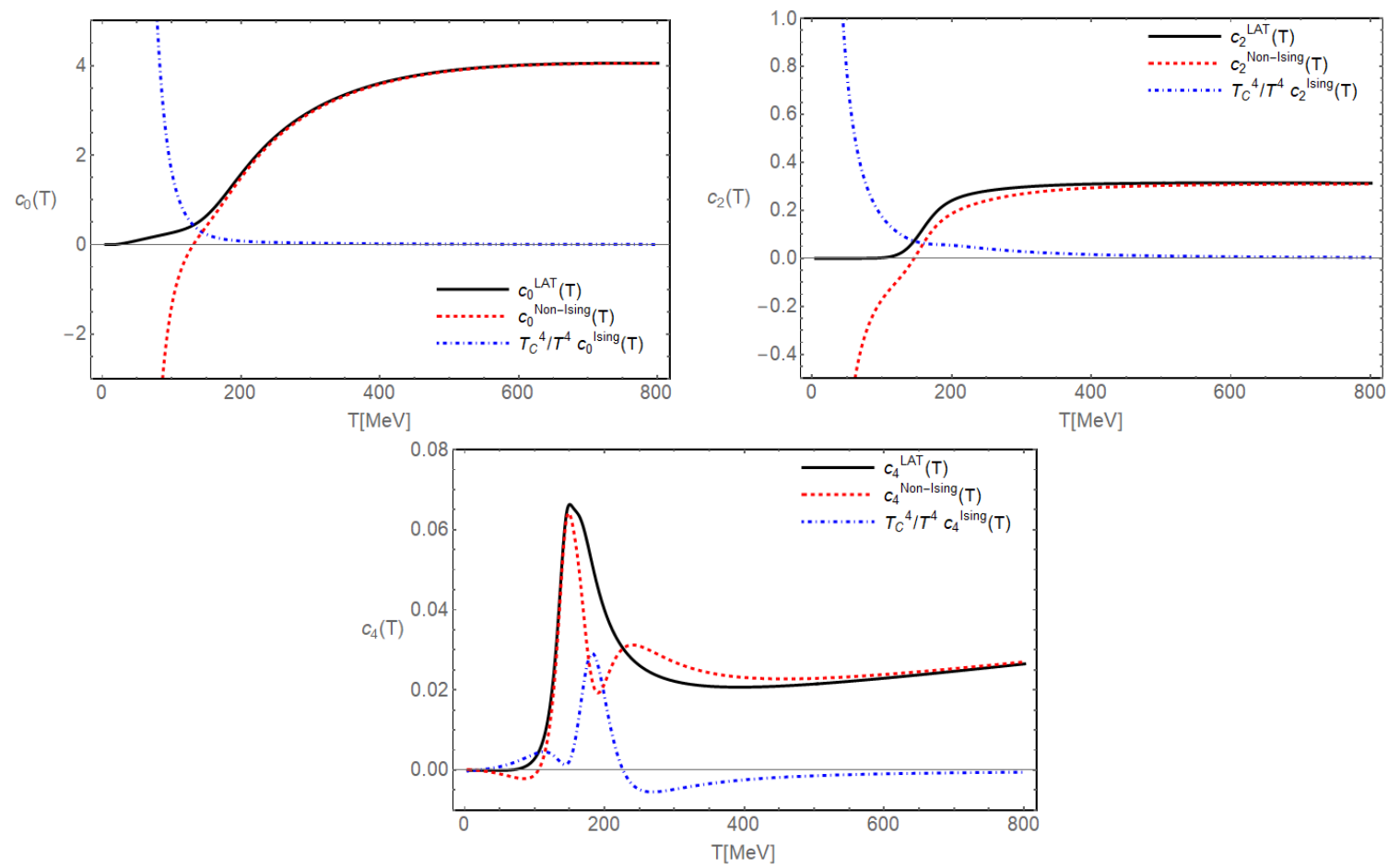

Figure 3: Comparison of critical (blue, dot-dashed) and "Non-Ising" (red, dashed) contributions to baryon susceptibilities up to $\mathscr{O}\left(\mu_{B}^{4}\right)$ with the parametrized lattice data (black, solid).

between the pressure from our procedure and the one from the HRG model is parallel to the chiral transition curve.

In order to complete the thermodynamic description of the finalized equation of state obtained in Eq. (4.1), we can compute various thermodynamic observables of interest. In addition to the pressure, we compute the entropy density, baryon density, energy density and speed of sound normalized by the correct power of the temperature:

$$
\begin{aligned}
& \frac{P\left(T, \mu_{B}\right)}{T^{4}}, \quad \frac{S\left(T, \mu_{B}\right)}{T^{3}}=\frac{1}{T^{3}}\left(\frac{\partial P}{\partial T}\right)_{\mu_{B}}, \frac{n_{B}\left(T, \mu_{B}\right)}{T^{3}}=\frac{1}{T^{3}}\left(\frac{\partial P}{\partial \mu_{B}}\right)_{T}, \\
& \frac{\varepsilon\left(T, \mu_{B}\right)}{T^{4}}=\frac{S}{T^{3}}-\frac{P}{T^{4}}+\frac{\mu_{B}}{T} \frac{n_{B}}{T^{3}}, \quad c_{s}^{2}\left(T, \mu_{B}\right)=\left(\frac{\partial P}{\partial \varepsilon}\right)_{S / n_{B}} .
\end{aligned}
$$

They are shown in Figs. 4 - 8. The effect of the critical point on the thermodynamic isentropes (trajectories at constant $S / n_{B}$ in the QCD phase diagram) is shown in Fig. 9. The effect of the critical point is clearly visible in the distortion of the isentropes at large chemical potentials. By requiring thermodynamic stability, i.e. positivity of pressure, entropy density, baryon density, energy density and speed of sound, and causality, i.e $c_{s}^{2}<1$, over the whole phase diagram, it is possible to reduce the range of acceptable parameters in the non-universal Ising $\mapsto$ QCD map. By keeping the location of the critical point fixed $\left(\mu_{B C}=350 \mathrm{MeV}, T_{C} \simeq 143 \mathrm{MeV}\right)$, as well as the orientation of the axes $\left(\alpha_{1} \simeq 3.85^{\circ}, \alpha_{2}-\alpha_{1}=90^{\circ}\right)$, we investigated the role of the scaling parameters $w, \rho$. In Fig. 10, we can see in red the points corresponding to pathological parameter choices, while the blue dots correspond to acceptable ones. We notice that, while most commonly specific parameter 


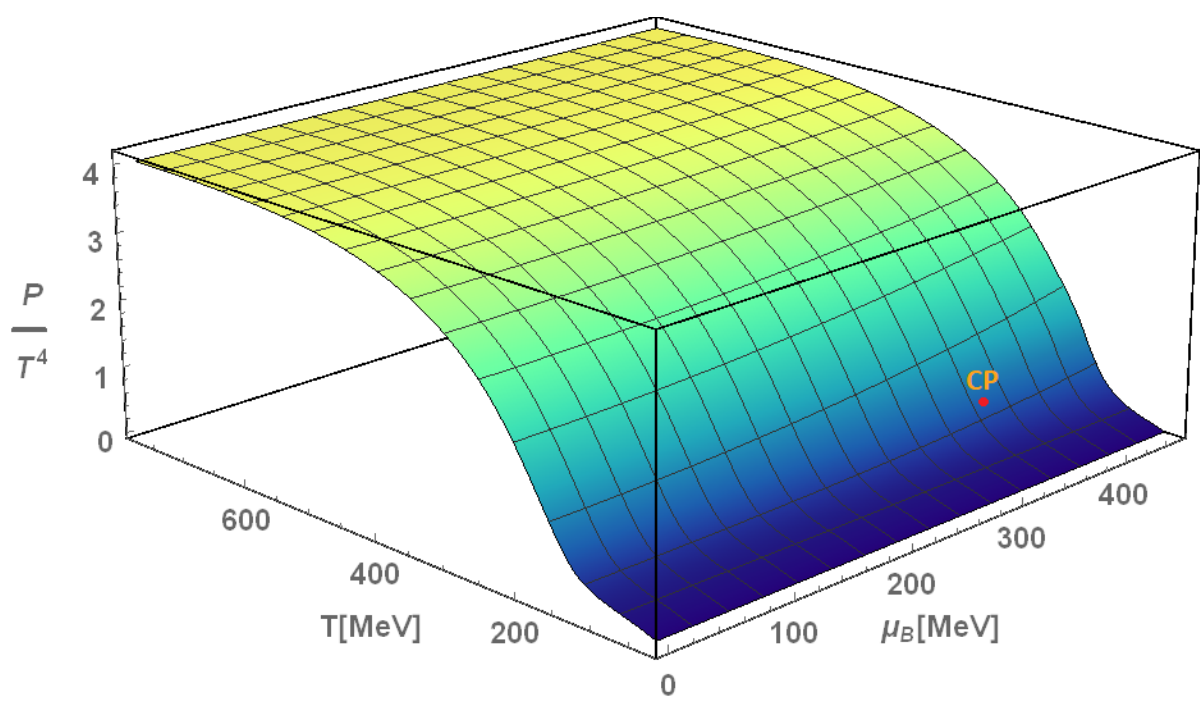

Figure 4: Pressure after merging with HRG.

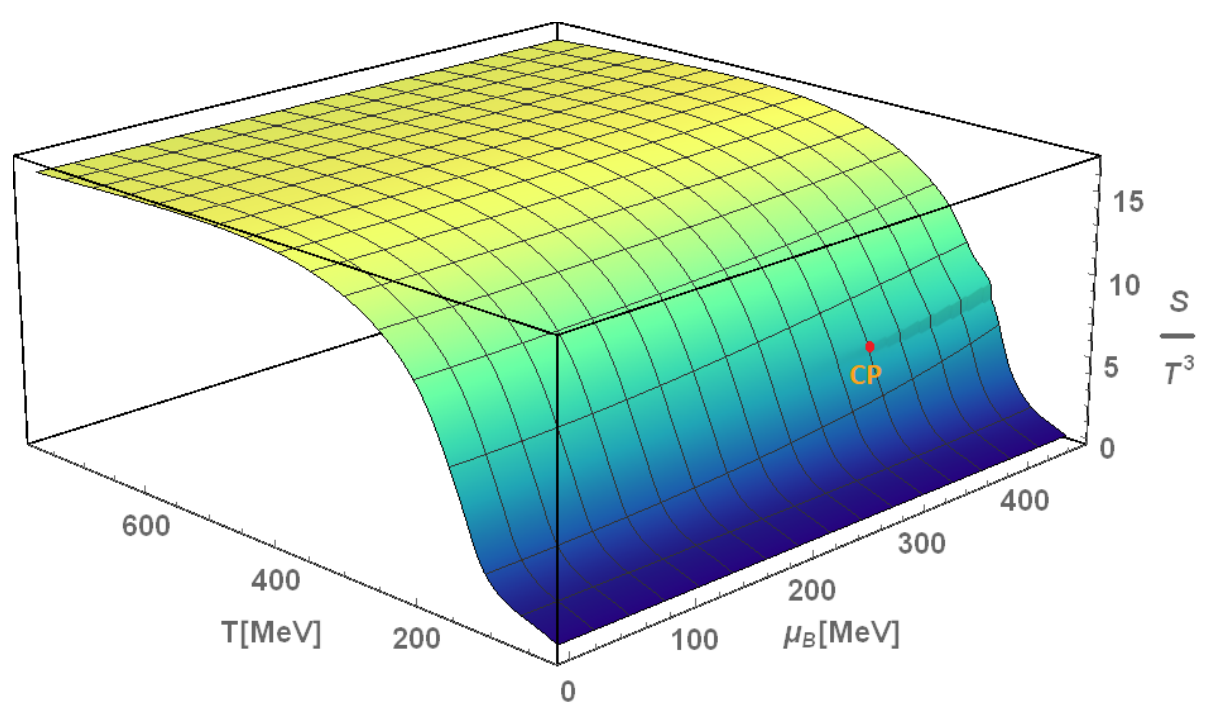

Figure 5: Entropy density after merging with HRG.

choices are unacceptable because of the negativity of $n_{B}$, for very low $w(w=0.25)$ we observe violation of causality as well $\left(c_{s}^{2}>1\right)$.

\section{Conclusions}

We presented a family of equations of state, which match available lattice QCD results up to $\mathrm{O}\left(\mu_{B}^{4}\right)$ and contain a critical point in the 3D Ising model universality class. These EoSs are meant to be used as an input into hydrodynamic simulations of the system created in heavy-ion collisions. A systematic scan of the parameter space, and relative comparison with experimental data from the BESII at RHIC, will hopefully allow us to constrain the size of the critical region and the location of the critical point. 


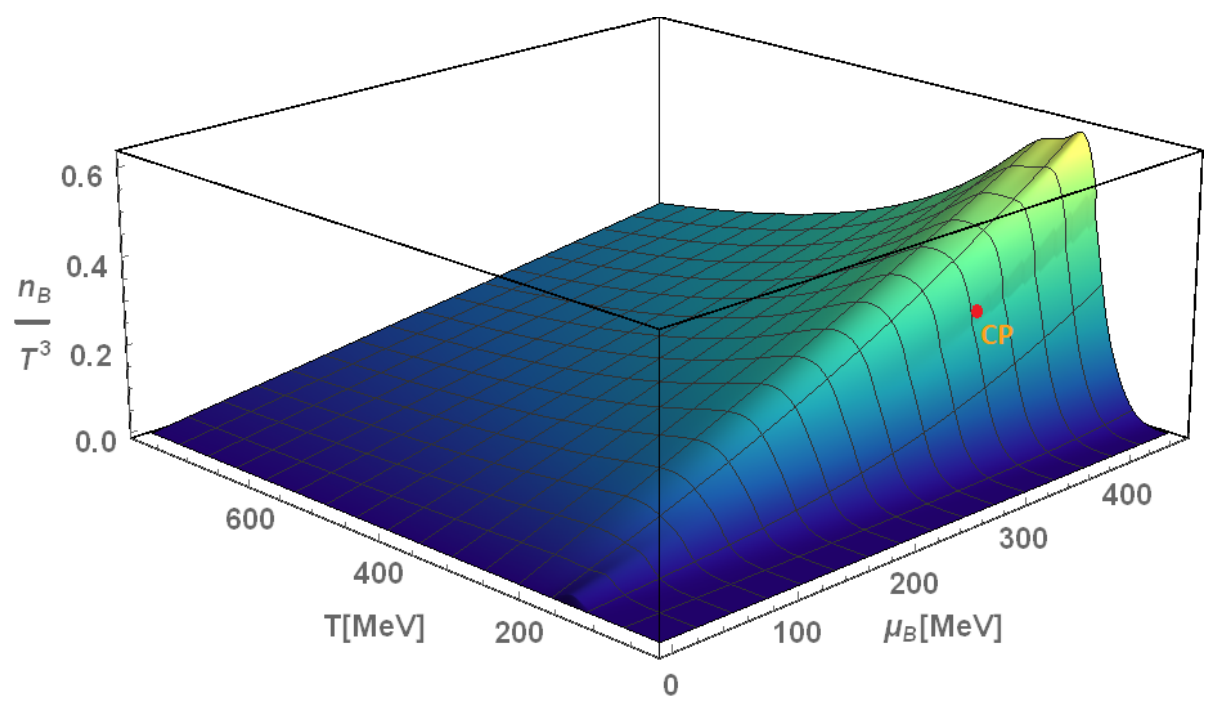

Figure 6: Baryon density after merging with HRG.

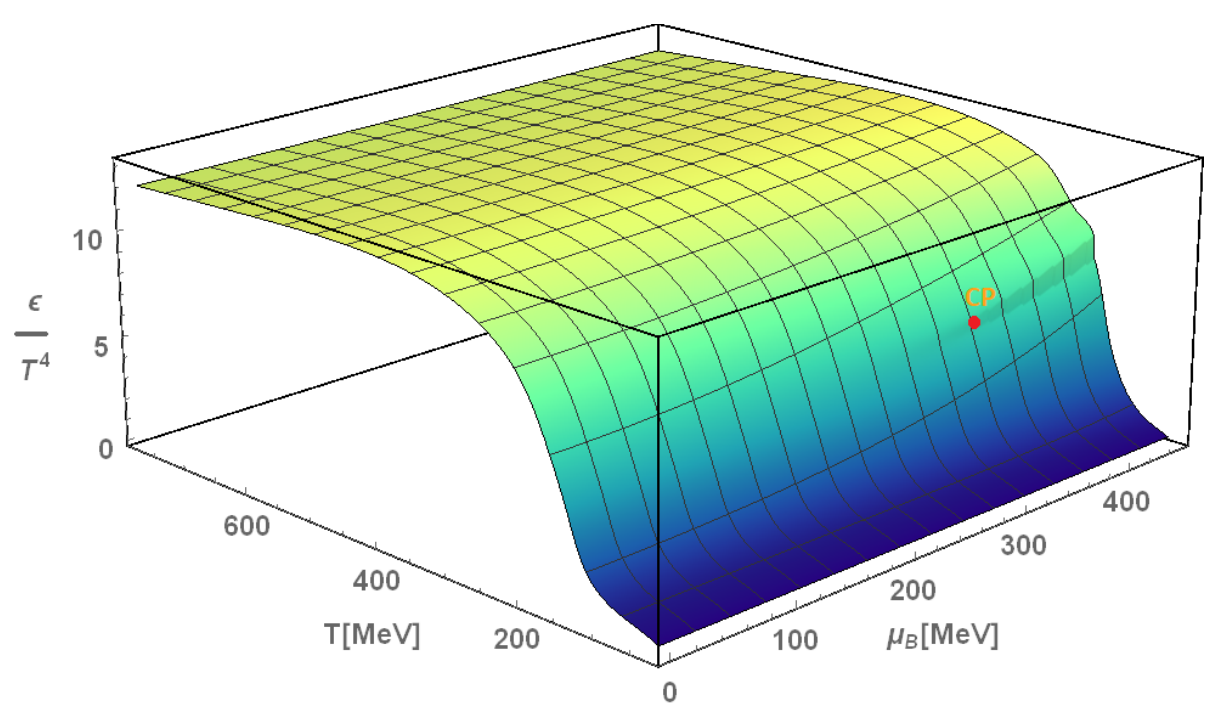

Figure 7: Energy density after merging with HRG.

\section{Acknowledgements}

This material is based upon work supported by the National Science Foundation under grant no. PHY-1654219 and by the U.S. Department of Energy, Office of Science, Office of Nuclear Physics within the framework of the Beam Energy Scan Theory (BEST) Topical Collaboration. The author also acknowledges the use of the Maxwell Cluster and the advanced support from the Center of Advanced Computing and Data Systems at the University of Houston.

\section{References}

[1] Aoki Y, Endrodi G, Fodor Z, Katz S D and Szabo K K 2006 Nature 443 675-678 (Preprint hep-lat/0611014) 


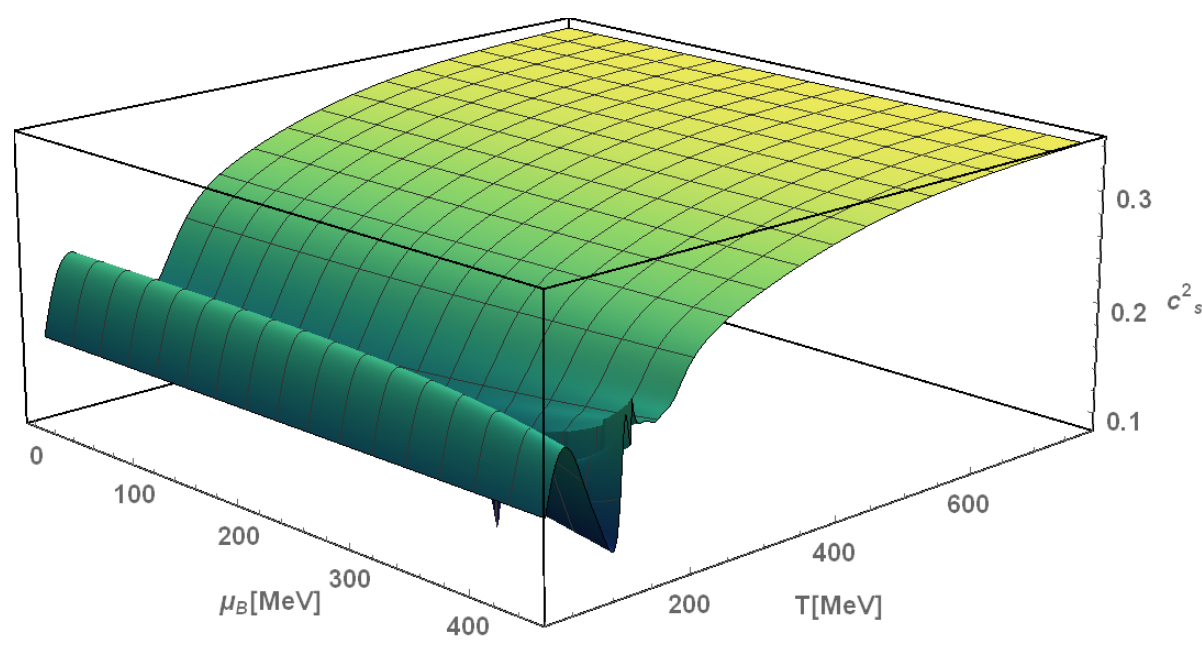

Figure 8: Speed of sound after merging with HRG.

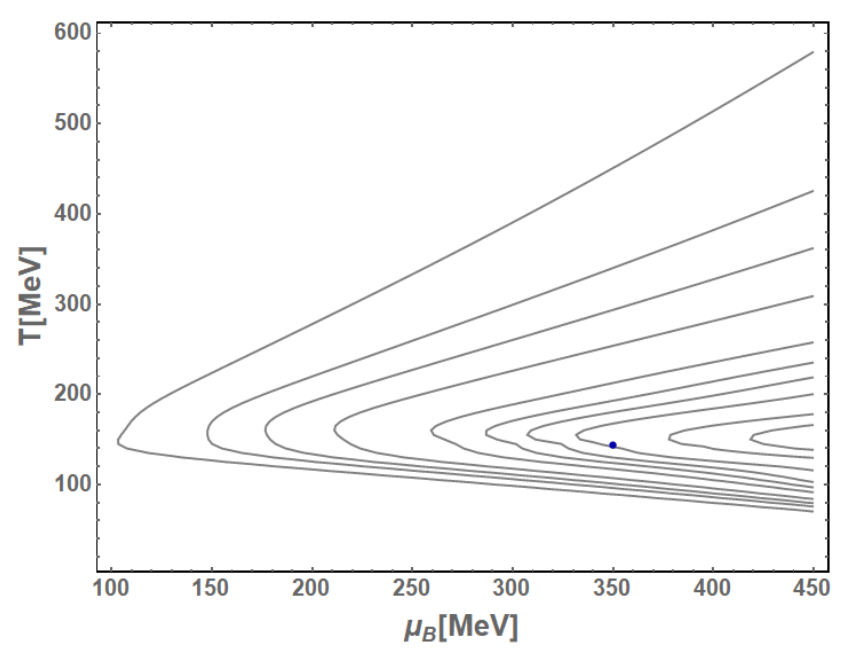

Figure 9: Lines of constant $S / n_{B}$ (from left to right $S / n_{B}=68,50,42,35,28,25,23,21,18,16$ ) in the QCD phase diagram. The blue dot indicates the location of the critical point.

[2] Stephanov M A 2006 PoS LAT2006 024 (Preprint hep-lat/ 0701002 )

[3] Stephanov M A 2010 Phys. Rev. D81 054012 (Preprint 0911.1772 )

[4] Nahrgang M, Leupold S, Herold C and Bleicher M 2011 Phys. Rev. C84 024912 (Preprint 1105.0622 )

[5] Stephanov M and Yin Y 2018 Phys. Rev. D98 036006 (Preprint 1712. 10305)

[6] Nahrgang M, Bluhm M, Schäfer T and Bass S A 2018 (Preprint 1804.05728)

[7] Parotto P, Bluhm M, Mroczek D, Nahrgang M, Noronha-Hostler J, Rajagopal K, Ratti C, Schäfer T and Stephanov M 2018 (Preprint 1805.05249)

[8] Borsanyi S, Endrodi G, Fodor Z, Jakovac A, Katz S D, Krieg S, Ratti C and Szabo K K 2010 JHEP 11077 (Preprint 1007.2580 ) 


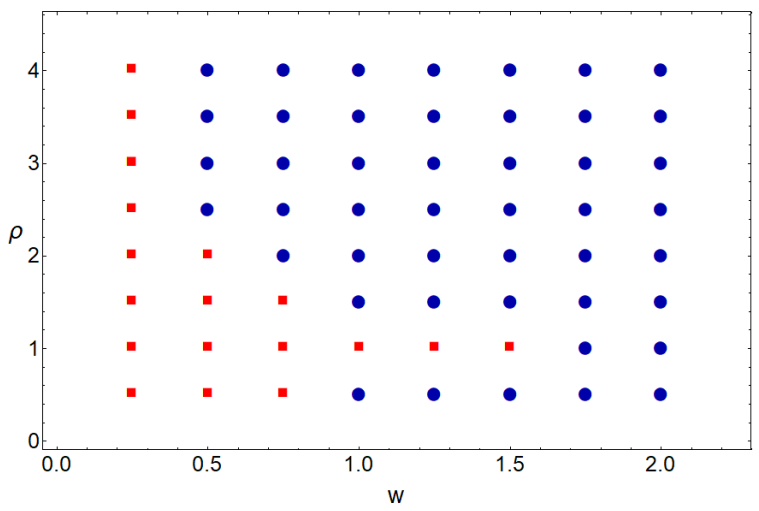

Figure 10: In red (squares) the points corresponding to pathological choices of parameters, in blue (dots) the acceptable ones.

[9] Borsanyi S, Fodor Z, Hoelbling C, Katz S D, Krieg S and Szabo K K 2014 Phys. Lett. B730 99-104 (Preprint 1309.5258$)$

[10] Bazavov A et al. (HotQCD) 2014 Phys. Rev. D90 094503 (Preprint 1407 . 6387)

[11] Borsanyi S et al. 2016 Nature 539 69-71 (Preprint 1606.07494 )

[12] Allton C R, Ejiri S, Hands S J, Kaczmarek O, Karsch F, Laermann E, Schmidt C and Scorzato L 2002 Phys. Rev. D66 074507 (Preprint hep-lat/ 0204010 )

[13] Allton C R, Doring M, Ejiri S, Hands S J, Kaczmarek O, Karsch F, Laermann E and Redlich K 2005 Phys. Rev. D71 054508 (Preprint hep-lat/ 0501030 )

[14] Gavai R V and Gupta S 2008 Phys. Rev. D78 114503 (Preprint 0806.2233 )

[15] Basak S et al. (MILC) 2008 PoS LATTICE2008 171 (Preprint 0910.0276 )

[16] Kaczmarek O, Karsch F, Laermann E, Miao C, Mukherjee S, Petreczky P, Schmidt C, Soeldner W and Unger W 2011 Phys. Rev. D83 014504 (Preprint 1011.3130)

[17] de Forcrand P and Philipsen O 2002 Nucl. Phys. B642 290-306 (Preprint hep-lat / 0205016 )

[18] D’Elia M and Lombardo M P 2003 Phys. Rev. D67 014505 (Preprint hep-lat/ 0209146 )

[19] Wu L K, Luo X Q and Chen H S 2007 Phys. Rev. D76 034505 (Preprint hep-lat/ 0611035 )

[20] D’Elia M, Di Renzo F and Lombardo M P 2007 Phys. Rev. D76 114509 (Preprint 0705.3814 )

[21] Conradi S and D'Elia M 2007 Phys. Rev. D76 074501 (Preprint 0707 . 1987)

[22] de Forcrand P and Philipsen O 2008 JHEP 11012 (Preprint 0808 . 1096)

[23] D’Elia M and Sanfilippo F 2009 Phys. Rev. D80 014502 (Preprint 0904 . 14 00)

[24] Moscicki J T, Wos M, Lamanna M, de Forcrand P and Philipsen O 2010 Comput. Phys. Commun. 181 1715-1726 (Preprint 0911.5682 )

[25] Borsanyi S, Endrodi G, Fodor Z, Katz S D, Krieg S, Ratti C and Szabo K K 2012 JHEP 08053 (Preprint 1204.6710)

[26] Hegde P (BNL-Bielefeld-CCNU) 2014 Nucl. Phys. A931 851-855 (Preprint 1408 . 6305)

[27] Gunther J, Bellwied R, Borsanyi S, Fodor Z, Katz S D, Pasztor A and Ratti C 2017 EPJ Web Conf. 13707008 (Preprint 1607.02493 ) 
[28] Bazavov A et al. 2017 Phys. Rev. D95 054504 (Preprint 1701.04325 )

[29] D’Elia M, Gagliardi G and Sanfilippo F 2017 Phys. Rev. D95 094503 (Preprint 1611.08285 )

[30] Borsanyi S, Fodor Z, Guenther J N, Katz S K, Szabó K K, Pasztor A, Portillo I and Ratti C 2018 (Preprint 1805.04445)

[31] Ratti C 2018 Rept. Prog. Phys. 81084301 (Preprint 1804 .07810)

[32] Nonaka C and Asakawa M 2005 Phys. Rev. C71 044904 (Preprint nucl-th/ 0410078 )

[33] Guida R and Zinn-Justin J 1997 Nucl. Phys. B489 626-652 (Preprint hep-th/9610223)

[34] Schofield P, Litster J D and Ho J T 1969 Phys. Rev. Lett. 23 1098-1102

[35] Bluhm M and Kampfer B 2006 PoS CPOD2006 004 (Preprint hep-ph / 0611083 )

[36] Rehr J J and Mermin N D 1973 Phys. Rev. A8 472-480

[37] Cea P, Cosmai L and Papa A 2016 Phys. Rev. D93 014507 (Preprint 1508 . 07599)

[38] Bonati C, D’Elia M, Mariti M, Mesiti M, Negro F and Sanfilippo F 2015 Phys. Rev. D92 054503 (Preprint 1507.03571)

[39] Bellwied R, Borsanyi S, Fodor Z, Günther J, Katz S D, Ratti C and Szabo K K 2015 Phys. Lett. B751 559-564 (Preprint 1507.07510)

[40] Patrignani C et al. (Particle Data Group) 2016 Chin. Phys. C40 100001

[41] Alba P et al. 2017 Phys. Rev. D96 034517 (Preprint 1702 . 01113)

[42] Bellwied R, Borsanyi S, Fodor Z, Katz S D, Pasztor A, Ratti C and Szabo K K 2015 Phys. Rev. D92 114505 (Preprint 1507.04627 ) 